\title{
Longterm Safety of Rituximab: Final Report of the Rheumatoid Arthritis Global Clinical Trial Program over 11 Years
}

\author{
Ronald F. van Vollenhoven, Roy M. Fleischmann, Daniel E. Furst, Stuart Lacey, \\ and Patricia B. Lehane
}

ABSTRACT. Objective. Final evaluation of the longterm safety of rituximab (RTX) in rheumatoid arthritis (RA) up to 11 years.

Methods. Pooled observed case analysis of data from patients with moderate to severe, active RA in a global clinical trial program.

Results. As of September 2012, 3595 patients received a mean of 4 courses (range 1-20) of RTX over 11 years $[14,816$ patient-years (PY)]. Of these, 1246 patients had $>5$ years of followup (8970 PY). A pooled placebo population $(n=818)$ was included in the analysis. The overall serious infection event (SIE) rate was 3.76/100 PY (2.71/100 PY in patients observed for $>5 \mathrm{yrs})$ and comparable with rates reported previously at 9.5 years (3.94/100 PY and 3.26/100 PY, respectively). SIE rates continued to be similar before and during/after development of low immunoglobulin levels, and serious opportunistic infections remained rare. Rates of cardiac events remained consistent with previous analysis and with rates in the general RA population. No increased risk of malignancy over time was observed. Conclusion. This final report demonstrates that RTX remains well tolerated over time and multiple courses. No new safety risks were identified and there was no increase in the rate of any types of adverse events with prolonged exposure to RTX during 11 years of observation. (First Release August 15 2015; J Rheumatol 2015;42:1761-6; doi:10.3899/jrheum.150051)

Key Indexing Terms:

RHEUMATOID ARTHRITIS

Rituximab (RTX), an anti-CD20 monoclonal antibody, has been shown to improve signs and symptoms of disease and reduce radiographic damage in patients with rheumatoid arthritis (RA) $)^{1,2}$. A previously published analysis found that exposure to multiple courses of RTX over 9.5 years of observation was associated with a well-tolerated safety profile ${ }^{3}$.

From the Unit for Clinical Therapy Research, Inflammatory Diseases, Karolinska Institute, Stockholm, Sweden; Department of Internal Medicine, University of Texas, Southwestern Medical Center, Dallas,

Texas; Department of Rheumatology, University of California, Los Angeles Medical Center, Los Angeles, California, USA; Roche Products Ltd. Welwyn Garden City, UK.

Sponsored and funded by F. Hoffmann-La Roche, Genentech (a member of the Roche group), and Biogen Idec. Support for third-party writing assistance for this manuscript, furnished by Vivian Chen, PharmD of Health Interactions, was funded by F. Hoffmann-La Roche Ltd.

R.F. van Vollenhoven, MD, Chief of the Unit for Clinical Therapy Research, Inflammatory Disease, Karolinska Institute; R.M. Fleischmann, MD, Clinical Professor of Medicine, Department of Internal Medicine, University of Texas, Southwestern Medical Center; D.E. Furst, MD, Carl M. Pearson Professor of Medicine, Department of Rheumatology, University of California, Los Angeles Medical Center; S. Lacey, MSc, Statistician, Roche Products Ltd.; P.B. Lehane, PhD, Senior Clinical Development Scientist, Roche Products Ltd.

Address correspondence to Professor R.F. van Vollenhoven, Unit for Clinical Therapy Research, Inflammatory Diseases, The Karolinska University Hospital, D1:00, 17176, Stockholm, Sweden.

E-mail: ronald.van.vollenhoven@ki.se

Full Release Article. For details see Reprints/Permissions at jrheum.org Accepted for publication June 17, 2015.

\section{RITUXIMAB SAFETY}

While these data are reassuring, continued monitoring of the longterm safety of RTX is necessary to understand the potential risk associated with prolonged and repeated peripheral $\mathrm{B}$ cell depletion. In particular, the potential cumulative risks of serious infection events (SIE) and malignancies require further followup. The objective of our analysis was to provide the final report on the longterm safety of RTX over 11 years of observation in the clinical trial program. It represents 2854 additional patient-years (PY) of followup with the inclusion of an additional 401 patients treated with RTX, pooled from an RTX RA open-label prospective study ${ }^{4}$. This update not only increases the probability of detecting uncommon and infrequent safety risks, but also affords rheumatologists a higher level of confidence in the data.

\section{MATERIALS AND METHODS}

Safety data were evaluated from patients with moderate to severe, active RA treated with RTX plus methotrexate (MTX) from a global clinical trial program ( 8 randomized clinical trials, 2 longterm open-label extensions, 1 open-label prospective study) $)^{1,4,5,6,7,8}$. Eligibility criteria and study designs have been previously presented ${ }^{1,5,6,7,8}$. Each RTX course consisted of either $2 \times 1000 \mathrm{mg}$ or $2 \times 500 \mathrm{mg}$ intravenous infusions, 2 weeks apart. Repeat treatment was based on the physician's decision of clinical need and included evidence of active disease (either swollen/tender joint counts $\geq 8$ or Disease Activity Score in 28 joints $\geq 2.6$ ). Safety assessments were conducted as previously reported ${ }^{3}$. The analysis consisted of the following populations: the RTX all-exposure population (all patients exposed to at least 1 or part of

Personal non-commercial use only. The Journal of Rheumatology Copyright @ 2015. All rights reserved. 
1 RTX infusion, regardless of dose), the RTX longterm population (a subset of the all-exposure population with $>5$ yrs followup from first RTX exposure), and the pooled placebo population (all patients receiving placebo during placebo-controlled and study followup periods). Our longterm followup was completed in September 2012; the final results are provided here.

\section{RESULTS}

Demographics. As of September 2012, 3595 patients received a mean of 4 courses (range 1-20) of RTX over 11 years, providing 14,816 PY of observation in the RTX all-exposure population. Of these patients, 1246 patients (8970 PY) were followed for > 5 years (RTX longterm population). The placebo population included 818 patients (1107 PY). Patients in the RTX longterm population had a longer mean RA disease duration and a greater number $(\mathrm{n}=$ 2.3) of previous disease-modifying antirheumatic drugs (excluding MTX) than the all-exposure and placebo populations. Baseline demographics and disease characteristics were otherwise similar across groups. The placebo population included patients with a substantially shorter mean duration of followup compared with the RTX populations ( $>50 \%$ withdrew from placebo followup by $1 \mathrm{yr}$ and $93 \%$ withdrew by 3 yrs).

The greatest number of withdrawals occurred following the first 2 courses (1034 patients, 29\%), principally for nonsafety reasons and because 2 studies limited patients to receiving 1 or 2 courses per protocol. Across the trials, there was a normal expected dropout rate (1-14\%) for subsequent courses. Overall, withdrawals due to adverse events (AE) were infrequent (241 patients, 7\%). Most withdrawals were attributed to "other" reasons that included insufficient therapeutic response, failure to return, violation of selection criteria at entry, other protocol violation, refused treatment/did not cooperate, withdrew consent, administrative/other (including patient entry to extension protocols), lost to followup, physician's decision to withdraw, and pregnancy. Data from the patients who remained in the study were largely complete with any missing data considered to be missing at random.

$A E$ and serious $A E(S A E)$. Rates of $\mathrm{AE}$ and $\mathrm{SAE}$ remained similar in the RTX all-exposure, RTX longterm, and placebo populations (Table 1). The rate of all AE over time was highest during the first 6 months after the first RTX exposure, in part because of infusion-related reactions (IRR) that predominantly occurred with the first infusion of the first course. Rates of AE and SAE subsequently decreased and remained stable thereafter, irrespective of the number of RTX courses received (Figure $1 \mathrm{~A}-1 \mathrm{~B}$ ). SAE that occurred in $>1 \%$ of patients receiving RTX were RA exacerbations $(n=83$, $2 \%)$, pneumonia $(\mathrm{n}=74,2 \%)$, osteoarthritis $(\mathrm{n}=55,2 \%)$, and falls $(n=62,2 \%)$.

There were 78 deaths in the all-exposure population $(0.53$ events/100 PY, 95\% CI 0.42-0.66) and 7 in the placebo population (0.63 events/100 PY, 95\% CI 0.30-1.33). Death rates were consistent with the previous analysis and with the rate expected in the general US population, adjusted for age and $\operatorname{sex}^{9}$. Frequent causes of death included malignancies $(n=19)$, cardiovascular events $(n=15)$, infections $(n=15)$, respiratory disorders $(n=8)$, and nervous system events $(n=$ 4), which are consistent with those expected in this RA population of biologic-treated patients. The incidence of death did not appear to increase over multiple courses and there have been no fatal IRR. There was no evidence of an increased rate of any type of fatal event with prolonged RTX exposure.

In the all-exposure population, 241 patients (7\%) withdrew prematurely from their respective study because of $\mathrm{AE}$ (including $\mathrm{SAE}$ ). The incidence of $\mathrm{AE}$ leading to withdrawal was highest in the first course and decreased thereafter with each treatment course. The most common types of AE leading to withdrawal were musculoskeletal and connective tissue disorders (mainly RA exacerbation), IRR, neoplasms (benign, malignant, and unspecified), and infections and infestations.

Infections. The overall rates of all infections and SIE changed very little from the previous analysis of this cohort and remained similar across analysis populations (Table 1). The rate of SIE generally remained stable over time (Figure 1C) and over multiple treatment courses (Figure 1D). A numerically higher SIE rate between Year 5 and Year 6 was not observed in subsequent years. Large CI were observed at $>9$ years because of low PY exposure. The most frequent SIE in the all-exposure population were lower respiratory tract infections, predominantly pneumonia $(2 \%)$.

Infections of interest in $R A$. Serious opportunistic infections remained rare (all-exposure, 0.05 events/100 PY; placebo, 0.09 events/100 PY). No additional serious opportunistic infections occurred since the 7 events previously reported ${ }^{3}$. A subanalysis of patients who went on to receive approved biologic agents for RA, namely tumor necrosis factor (TNF) inhibitors or abatacept, showed that the use of subsequent biologics after RTX therapy was not associated with an increased SIE rate (Table 2). Although limited by small patient numbers, SIE rates were similar in patients who received their biologic $<6$ months $(\mathrm{n}=98)$ and $\geq 6$ months $(\mathrm{n}=255)$ after last RTX dose (3.65 events/100 PY, 95\% CI $1.64-8.13$ vs $4.28,95 \%$ CI 2.53-7.22).

Screening for latent tuberculosis (TB; by means of purified protein derivative testing) in the RTX clinical development program was not mandated by protocol, although it can be assumed that patients who received a prior TNF inhibitor would have undergone such screening. Two cases of pulmonary TB, treated with anti-TB medication, occurred in the all-exposure population and both were reported previously $^{3}$. No cases were observed of extrapulmonary TB, atypical mycobacterial infection, or multidrug-resistant TB.

One case of de novo hepatitis B was reported previously ${ }^{1}$. Within the RTX RA clinical development program, there

Personal non-commercial use only. The Journal of Rheumatology Copyright $\subset$ $\subset$ 2015. All rights reserved 
Table 1. Summary of AE rates per $100 \mathrm{PY}$.

\begin{tabular}{lccc}
\hline Variables & $\begin{array}{c}\text { RTX All-exposure, } \\
\mathrm{n}=3595\end{array}$ & $\begin{array}{c}\text { RTX Longterm, }>5 \text { Yrs, } \\
\mathrm{n}=1246\end{array}$ & $\begin{array}{c}\text { Pooled Placebo, } \\
\mathrm{n}=818\end{array}$ \\
\hline Exposure, PY & 14,816 & 8970 & 1107 \\
AE rate (95\% CI) & $239.11(236.63-241.61)$ & $219.36(216.31-222.44)$ & $315.43(305.14-326.06)$ \\
SAE rate (95\% CI) & $13.82(13.24-14.43)$ & $11.88(11.19-12.62)$ & $13.82(11.79-16.19)$ \\
Infection rate (95\% CI) & $75.70(74.31-77.11)$ & $70.52(68.81-72.28)$ & $90.39(84.96-96.17)$ \\
SIE rate (95\% CI) & $3.76(3.46-4.09)$ & $2.71(2.39-3.07)$ & $3.79(2.80-5.13)$ \\
\hline
\end{tabular}

AE: adverse event; PY: patient-years; RTX: rituximab; SAE: serious adverse events; SIE: serious infection events.

were no reported cases of hepatitis $\mathrm{B}$ viral reactivation (patients with active hepatitis B infection, defined by the presence of HBsAg, were excluded from the RA clinical trials; however, patients who tested negative for HBsAg and hepatitis B DNA but positive for hepatitis B core antigen were included). As of the data cutoff, 131 (6.5\%) of 2096 evaluable patients in the all-exposure population were enrolled with a positive test for hepatitis B core antigen. This group of patients received up to 16 courses of RTX. The overall proportions of these patients who experienced AE, $\mathrm{SAE}$, or liver function test abnormalities were consistent with those in the all-exposure population, and there were no liver-related SAE.

Infection risk in patients with low immunoglobulin (Ig) levels. Depending on study protocols, Ig serum levels were measured every 8-16 weeks throughout treatment periods. Mean Ig concentrations decreased from baseline over time and over courses. The largest decreases were observed in IgM levels. At pre-RTX Day 1 baseline, few patients $(\leq 2.2 \%)$ from the RTX all-exposure population had total $\operatorname{Ig}, \operatorname{IgA}, \operatorname{IgG}$, or IgM concentrations below the lower limit of normal (LLN). After RTX treatment (postbaseline), $\leq 3.9 \%$ had IgA or total Ig levels below LLN at any time, while $14.8 \%$ had IgG levels < LLN and 37.9\% had IgM levels $<$ LLN at any time. The proportion of patients with IgM but not IgG or IgA below the LLN continued to increase over the first 5 RTX courses (data not shown). The clinical relevance of low Ig serum levels and potential to predispose individuals to infections was evaluated. An analysis was performed to assess the rates of serious infections in patients before and after a low $(<\mathrm{LLN}) \operatorname{IgG}$ or IgM level for at least a 4-month period (or 2 consecutive study visits). Out of 3595 patients (24\%), 863 developed IgM $<$ LLN for at least 4 months while 143/3595 (4\%) developed IgG < LLN for at least 4 months. For both of these Ig classes, SIE rates per $100 \mathrm{PY}$ were similar before (IgM 2.80, 95\% CI 2.09-3.75; IgG 6.75, 95\% CI 4.48-10.15) and during/after development of low $\operatorname{Ig}(\operatorname{IgM} 3.84,95 \%$ CI 3.20-4.62; $\operatorname{IgG} 8.16,95 \%$ CI 5.86-11.36).

SIE rates in patients who developed low IgG levels were higher than corresponding rates in patients who never developed low $\operatorname{IgG}$ and higher than those in the all-exposure population, both before and after development of low IgG levels, suggesting that these patients may have a higher inherent risk of developing SIE. Limitations of the Ig analysis included low patient numbers in the IgG subgroup $(n=143)$, lack of a placebo comparator, and lack of correlation between SIE onset and recording of low Ig levels.

Cardiac events. Myocardial infarction (MI) was the most frequent cardiac AE (58 events in 52 patients). Most affected patients had $\geq 1$ risk factor for MI. The rate of MI in patients receiving RTX ( 0.39 events/100 PY, 95\% CI 0.30-0.51) was consistent with rates in the general RA population $(0.48,95 \%$ CI $0.34-0.94$ to 0.59 events/100 PY, 95\% CI 0.37-0.61) ${ }^{10}$.

Malignancies. There was no evidence of an increased risk of malignancy of any type over time or RTX course (exclusion criteria for study entry included history of prior malignancy). In the all-exposure population, there were 109 confirmed malignancies (0.74 events/100 PY, 95\% CI 0.60-0.88), excluding nonmelanoma skin cancers (NMSC) and nonmalignant events. These rates were comparable with rates observed in the placebo population ( 0.81 events/100 PY) and those in the general RA population $\left(1.17 / 100 \mathrm{PY}^{11}\right.$ and 1.30/100 PY, 95\% CI 1.19-1.41 ${ }^{12}$ ). A total of 68 NMSC $(0.46 / 100 \mathrm{PY})$ were reported. For solid tumors, the most frequently reported malignancy was breast cancer (16 events, $0.14 / 100$ PY, 95\% CI 0.08-0.22 in female patients only), comparable with or lower than RA observational studies (0.13/100 PY $\mathrm{PY}^{11}$ and $0.21 / 100 \mathrm{PY}, 95 \%$ CI $\left.0.17-0.26^{12}\right)$. Age- and sex-matched standardized incidence ratios (SIR) for all confirmed malignancies did not indicate an increased risk of malignancy for patients receiving RTX with RA (1.07, 95\% CI 0.88-1.29) compared with the general US population (SEER database ${ }^{13}$ ) and compared with published data in adults with RA $\left(1.05,95 \%\right.$ CI $\left.1.01-1.09^{14}\right)$. The SIR of breast cancer for patients with RA receiving RTX $(0.63,95 \%$ CI 0.36-1.03) was also comparable with the published data in adults with RA $\left(0.84,95 \%\right.$ CI $\left.0.79-0.90^{14}\right)$. The observed incidences of all malignancies (overall and by type) in RTX clinical trials were (based on reported data in the literature) within the expected ranges for the RA population.

\section{DISCUSSION}

We report here the final results of the global RTX clinical trial program in RA. These longterm followup data confirm

Personal non-commercial use only. The Journal of Rheumatology Copyright (C) 2015. All rights reserved. 


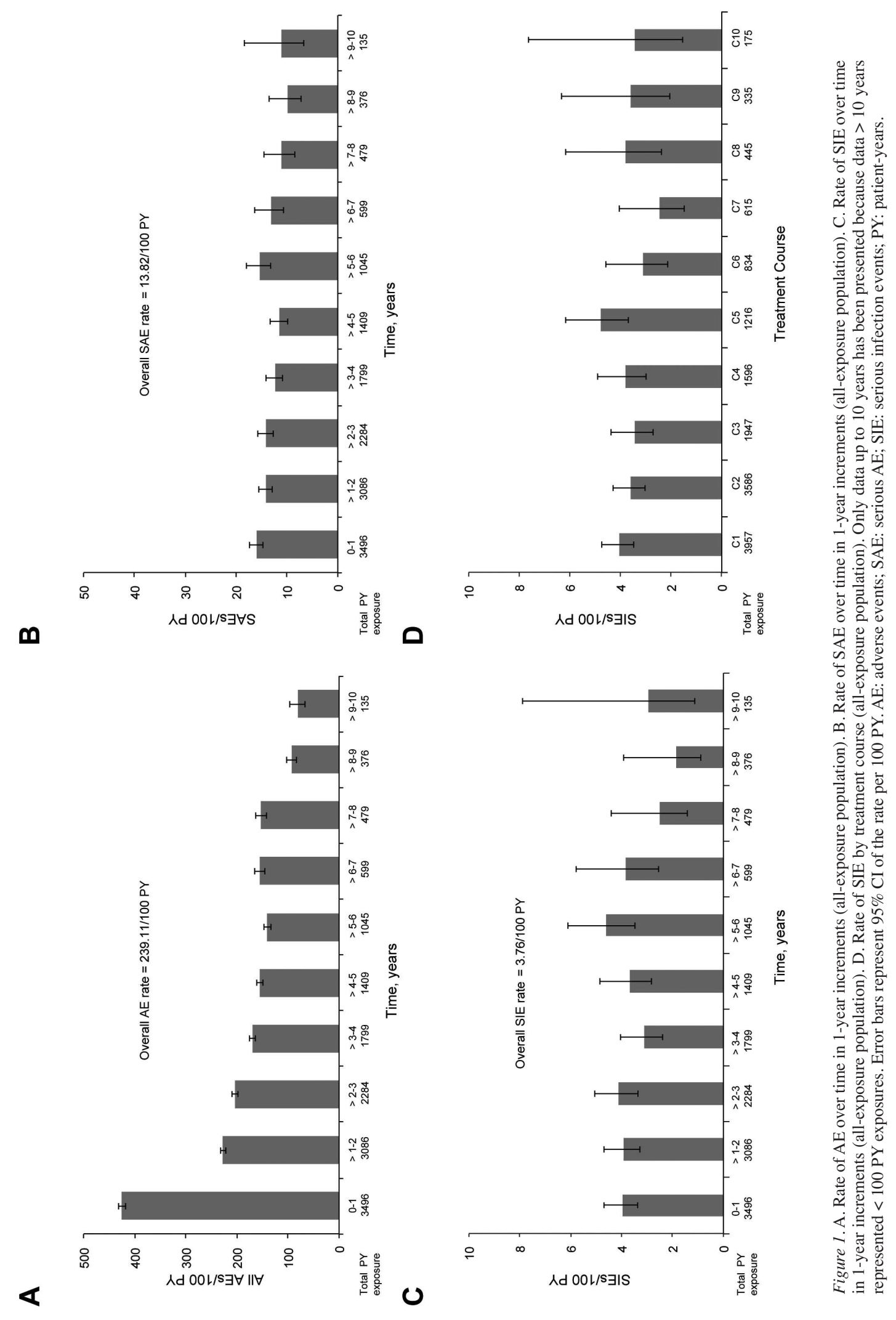

Personal non-commercial use only. The Journal of Rheumatology Copyright $\odot$ 2015. All rights reserved. 
Table 2. SIE rates before and after treatment with biologics, including TNF inhibitors. Multiple occurrences of the same event in 1 individual are counted multiple times. DMARD received after the first day of study RTX dose are the DMARD of interest. N is the number of patients receiving treatment with a DMARD post-RTX.

Variables
All Patients Receiving Any Biologic Following RTX Treatment, $\mathrm{n}=353$

Before Other Biologic, During RTX Treatment
After Other Biologic, After RTX Treatment
Subset of Patients Receiving a TNF Inhibitor Following RTX Treatment, $\mathrm{n}=280$

Before TNF Inhibitor, After Other TNF Inhibitor, During RTX Treatment After RTX Treatment

\begin{tabular}{lccc}
\hline Total exposure, PY & 727.48 & 491.61 & 514.45 \\
Serious infections, $\mathrm{n}$ & 32 & 20 & 22 \\
Serious infections/100 PY & 4.40 & 4.07 & 4.28 \\
$95 \%$ CI & $3.11-6.22$ & $2.62-6.31$ & 3.61 \\
\hline
\end{tabular}

SIE: serious infection events; TNF: tumor necrosis factor; DMARD: disease-modifying antirheumatic drugs; RTX: rituximab; PY: patient-years.

that RTX has a consistent safety profile over time and multiple courses up to 11 years of observation. While the number of patients in this update $(n=3595)$ did not significantly increase from what was previously reported $(\mathrm{n}=$ 3194), the total exposure time increased significantly by 2854 additional PY, representing a total followup time of 14,816 PY. An additional 619 patients more than the previous analysis were observed for $>5$ years, with some having received up to 20 RTX courses over 11 years. No new safety signals or increased reporting rates of any types of AE were observed with increasing duration of exposure or courses of treatment. Rates of all AE were highest during the first 6 months, owing in part to IRR. Rates of AE and SAE subsequently decreased and remained stable thereafter, irrespective of the number of RTX courses received. Rates of all infections and serious infections remained similar to the previous analysis despite the increased observation time and additional courses.

Serious opportunistic infections remained rare. No additional cases of confirmed progressive multifocal leukoencephalopathy (PML) in the RA clinical trial program have been reported other than the single case described in $2008^{15}$. The occurrence of confirmed PML from spontaneous reporting and clinical trial sources remains very rare (8 confirmed cases in 271,615 patients treated with RTX, based on patient market exposure estimates as of November 2013). Thus, the reporting rate of confirmed PML in patients with RA is 2.95 (95\% CI 1.27-5.8) per 100,000 patients, which has remained stable over time (Roche data on file). Unreported cases of PML are not included in this calculation, which underscores the importance of physician vigilance and reporting of PML cases to most accurately estimate its prevalence. Although there were no reported cases of hepatitis B viral reactivation within the RA clinical development program, hepatitis B virus screening may be considered for high-risk patients before initiation of treatment with RTX. Use of subsequent biologics, including TNF inhibitors, in patients with RA previously treated with RTX was not associated with an increased SIE rate. Rates of MI and malignancies (overall and by type) continued to be consistent with those observed in the placebo population and in epidemiologic data from other RA cohorts.

These findings indicate that repeated peripheral B cell depletion with RTX did not give rise to any increased safety risk over time or increased reporting rates of any types of $\mathrm{AE}$ (including serious infections, cardiovascular events, malignancies, or fatal AE) in the global RA clinical trial program. With the exceptions of IRR and low Ig concentrations, the overall safety profile of RTX remains similar to that of the placebo population and consistent with published data for moderate to severe RA and with previous analyses of this patient cohort.

\section{REFERENCES}

1. Cohen SB, Emery P, Greenwald MW, Dougados M, Furie RA, Genovese MC, et al; REFLEX Trial Group. Rituximab for rheumatoid arthritis refractory to anti-tumor necrosis factor therapy: results of a multicenter, randomized, double-blind, placebo-controlled, phase III trial evaluating primary efficacy and safety at twenty-four weeks. Arthritis Rheum 2006;54:2793-806.

2. Keystone E, Emery P, Peterfy CG, Tak PP, Cohen S, Genovese MC, et al. Rituximab inhibits structural joint damage in patients with rheumatoid arthritis with an inadequate response to tumour necrosis factor inhibitor therapies. Ann Rheum Dis 2009;68:216-21.

3. van Vollenhoven RF, Emery P, Bingham CO 3rd, Keystone EC, Fleischmann RM, Furst DE, et al. Long-term safety of rituximab in rheumatoid arthritis: 9.5-year follow-up of the global clinical trial programme with a focus on adverse events of interest in RA patients. Ann Rheum Dis 2013;72:1496-502.

4. Loveless J, Olech E, Pritchard CH, Chai A, Kelman A, Klearman M. An open-label, prospective study (SUNDIAL) of the safety of rituximab in combination with disease-modifying anti-rheumatic drugs in patients with active rheumatoid arthritis (SUNDIAL). Arthritis Rheum 2009;60 Suppl 10:1660.

5. Emery P, Fleischmann R, Filipowicz-Sosnowska A, Schechtman J, Szczepanski L, Kavanaugh A, et al; DANCER Study Group. The efficacy and safety of rituximab in patients with active rheumatoid arthritis despite methotrexate treatment: results of a phase IIB randomized, double-blind, placebo-controlled, dose-ranging trial. Arthritis Rheum 2006;54:1390-400.

6. Keystone E, Fleischmann R, Emery P, Furst DE, van Vollenhoven $\mathrm{R}$, Bathon J, et al. Safety and efficacy of additional courses of rituximab in patients with active rheumatoid arthritis: an open-label extension analysis. Arthritis Rheum 2007;56:3896-908.

7. Emery P, Deodhar A, Rigby WF, Isaacs JD, Combe B, Racewicz AJ,

Personal non-commercial use only. The Journal of Rheumatology Copyright (C) 2015. All rights reserved. 
et al. Efficacy and safety of different doses and retreatment of rituximab: a randomised, placebo-controlled trial in patients who are biological naive with active rheumatoid arthritis and an inadequate response to methotrexate (Study Evaluating Rituximab's Efficacy in MTX iNadequate rEsponders (SERENE)). Ann Rheum Dis 2010;69:1629-35.

8. Tak PP, Rigby WF, Rubbert-Roth A, Peterfy CG, van Vollenhoven RF, Stohl W, et al; IMAGE Investigators. Inhibition of joint damage and improved clinical outcomes with rituximab plus methotrexate in early active rheumatoid arthritis: the IMAGE trial. Ann Rheum Dis 2011;70:39-46.

9. Centers for Disease Control and Prevention. National Vital Statistics Reports. Deaths: final data for 2005. [Internet. Accessed June 30, 2015.] Available from: www.cdc.gov/nchs/data/nvsr/nvsr56/ nvsr56_10.pdf

10. Dixon WG, Watson KD, Lunt M, Hyrich KL; British Society for Rheumatology Biologics Register Control Centre Consortium, Silman AJ, et al. Reduction in the incidence of myocardial infarction in patients with rheumatoid arthritis who respond to anti-tumor necrosis factor alpha therapy: results from the British Society for Rheumatology Biologics Register. Arthritis Rheum 2007;56:2905-12.

11. Mellemkjaer L, Linet MS, Gridley G, Frisch M, Møller H, Olsen JH. Rheumatoid arthritis and cancer risk. Eur J Cancer 1996;32A:1753-7.

12. Wolfe F, Michaud K. Biologic treatment of rheumatoid arthritis and the risk of malignancy: analyses from a large US observational study. Arthritis Rheum 2007;56:2886-95.

13. National Cancer Institute. Surveillance, epidemiology, and end results. [Internet. Accessed June 30, 2015.] Available from: seer.cancer.gov

14. Smitten AL, Simon TA, Hochberg MC, Suissa S. A meta-analysis of the incidence of malignancy in adult patients with rheumatoid arthritis. Arthritis Res Ther 2008;10:R45.

15. Fleischmann RM. Progressive multifocal leukoencephalopathy following rituximab treatment in a patient with rheumatoid arthritis. Arthritis Rheum 2009;60:3225-8. 